\title{
Quantitative Analysis of Immune-Reactive Cells among Leukocytes Is Useful for the Diagnosis of Drug Eruptions Caused by Bexarotene
}

\author{
Hiromu Chiba Yumi Kambayashi Kentaro Ohuchi Ryo Amagai \\ Erika Tamabuchi Akira Hashimoto Taku Fujimura \\ Department of Dermatology, Tohoku University Graduate School of Medicine, Sendai, \\ Japan
}

\author{
Keywords \\ Cutaneous T-cell lymphoma - Bexarotene - Drug eruption · Quantitative analysis of tumor- \\ infiltrating lymphocytes
}

\begin{abstract}
Bexarotene is a third-generation retinoid $\mathrm{X}$ receptor-selective retinoid that has been approved for use in the treatment of cutaneous T-cell lymphomas (CTCLs). Since the objective response rate of bexarotene is relatively high, with no racial differences, bexarotene can be administered to patients with phototherapy-resistant early CTCL as one of the first-line therapies in real-world clinical practice. Although various adverse events caused by bexarotene have been reported, there have been no reports of drug eruptions caused by bexarotene. One of the possible reasons is that it is difficult to distinguish a drug eruption from recurrence of $\mathrm{CTCL}$, histologically. In this report, drug eruptions in 2 patients with $\mathrm{CTCL}$ treated with bexarotene diagnosed by quantitative analysis of immunohistochemical staining by digital microscopy are described.
\end{abstract}

\section{Karger $\stackrel{N}{\%}$}


Chiba et al.: Drug Eruption by Bexarotene

\section{Introduction}

Cutaneous T-cell lymphomas (CTCLs), such as mycosis fungoides (MF), generally progress slowly from early to advanced stages [1]. Recently, several immunomodulatory drugs have become available for the treatment of CTCLs that are resistant to local skin therapies [2]. Among them, bexarotene is used as one of the most promising anti-CTCL drugs in first-line systemic therapy [2]. Since CTCL is a hematopoietic malignancy of lymphocyte origin [1], unlike solid tumors, it is difficult to distinguish tumor cells from tumor-infiltrating lymphocytes (TILs). In this report, drug eruptions occurring in 2 patients with CTCL treated with bexarotene are described.

\section{Case 1}

A 70-year-old Japanese man visited our outpatient clinic with a 9-year history of erythema on the trunk and extremities. He had been treated with topical steroid and narrow-band ultraviolet B for early-stage MF (T1bN0M0B0 stage I). On his visit, he had developed an infiltrated erythematous plaque on the trunk and extremities (Fig. 1a), though his phototherapy and topical therapy were continued. A biopsy specimen from the left side of the abdomen showed band-like infiltration of atypical lymphocytes with epidermotropism in the superficial dermis (Fig. 1b). Immunohistochemical staining showed that these atypical lymphocytes were positive for CD2, CD3, CD4, CD45, and CCR4 and negative for CD5, CD7, CD8, and CD30. Positron emission tomography-CT at the initial visit showed no evidence of lymph node or visceral involvement. The diagnosis was MF (T2N0M0B0 stage IIA), and he was administered oral bexarotene $\left(300 \mathrm{mg} / \mathrm{m}^{2}\right)$. Eight days after the administration of bexarotene, he had developed symmetrical, infiltrated, and disseminated erythema on the trunk and extremities with severe pruritus (Fig. 1c). A biopsy specimen from the newly arising eruption showed superficial perivascular infiltration of lymphocytes with prominent eosinophils (Fig. 1d). The diagnosis was a drug eruption caused by bexarotene. With oral prednisolone $30 \mathrm{mg} /$ day, his eruption disappeared, rapidly.

\section{Case 2}

A 66-year-old Japanese woman visited our outpatient clinic with erythematous nodules on the face, trunk, and extremities (Fig. 2a). A biopsy specimen showed dense infiltration of atypical lymphocytes without epidermotropism throughout the dermis (Fig. 2b). Immunohistochemical staining showed that these atypical lymphocytes were positive for CD2, CD3, CD4, CD7, and CCR4 and negative for CD5, CD8, and CD30. Positron emission tomography-CT at the initial visit showed no evidence of lymph node or visceral involvement. The diagnosis was primary cutaneous peripheral T-cell lymphoma not otherwise specified (T1N0M0B0 stage I), and she was treated with oral bexarotene (300 $\mathrm{mg} / \mathrm{m}^{2}$ ). Nine days after the administration of bexarotene, she developed symmetrical, infiltrated, and disseminated erythema on the trunk and extremities with severe pruritus (Fig. 2c). A biopsy specimen from a newly arising eruption showed superficial perivascular infiltration of lymphocytes with prominent eosinophils (Fig. 2d). The diagnosis was a drug eruption caused by bexarotene. With oral prednisolone $30 \mathrm{mg} /$ day, her eruption disappeared rapidly.

\section{Karger'}




\section{Case Reports in Oncology}

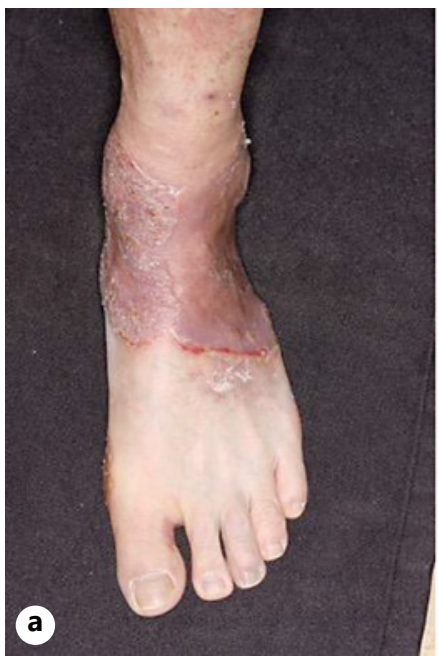

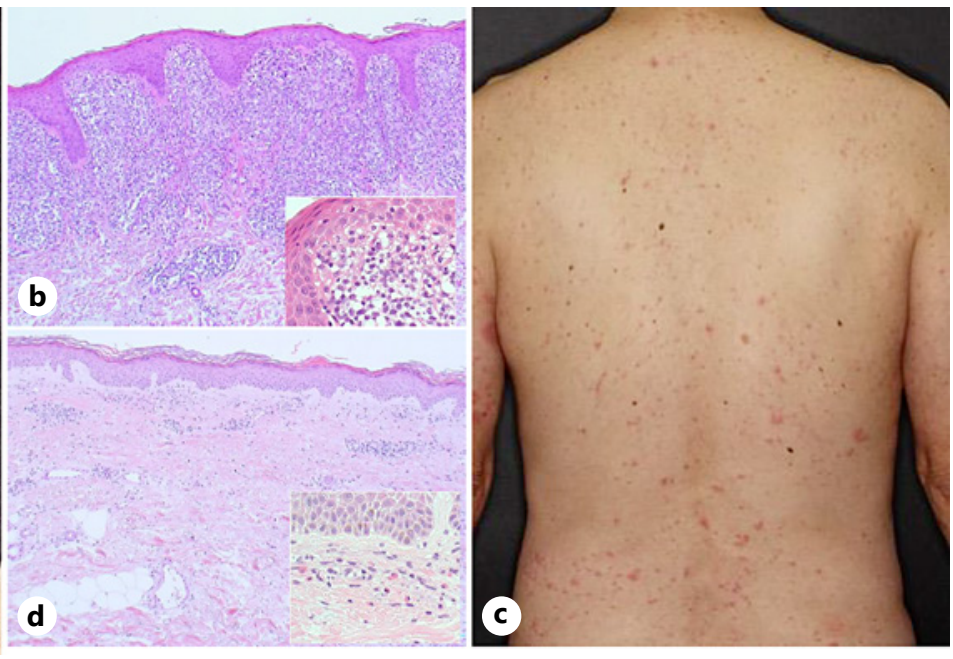

Fig. 1. a Case 1: an infiltrated erythematous plaque on the ankle. b A band-like infiltration of atypical lymphocytes with epidermotropism in the superficial dermis. $\mathbf{c}$ Eight days after the administration of bexarotene: symmetric, infiltrated, and disseminated erythema on the trunk and extremities with severe pruritus. $\mathbf{d}$ A biopsy specimen from the drug eruption: superficial perivascular infiltration of lymphocytes with prominent eosinophils.

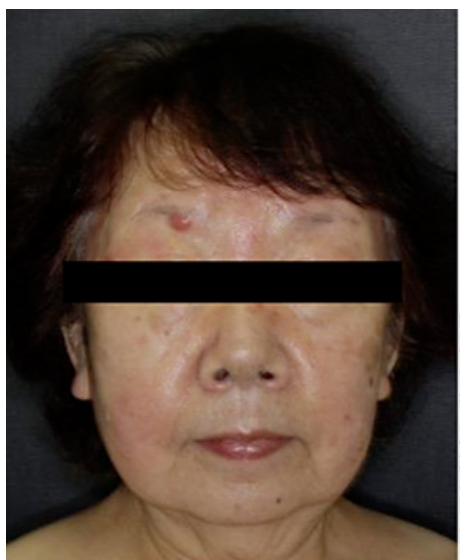

a

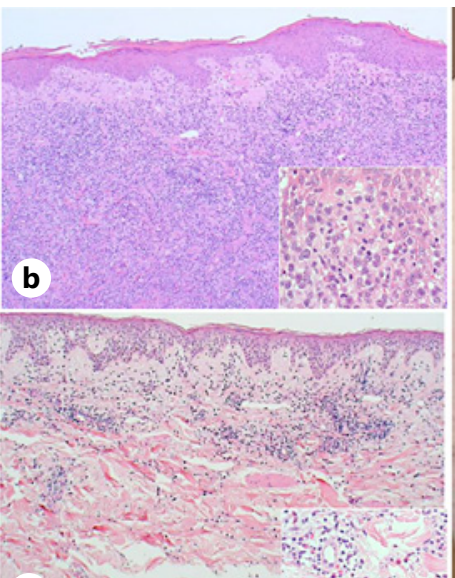

d

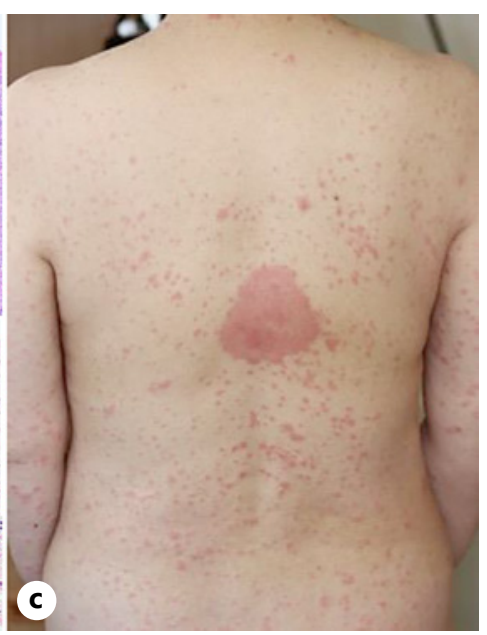

Fig. 2. a Case 2: erythematous nodules on the face. b Dense infiltration of atypical lymphocytes without epidermotropism throughout the dermis. c Nine days after the administration of bexarotene: symmetric, infiltrated, and disseminated erythema on the trunk and extremities with severe pruritus. $\mathbf{d}$ A biopsy specimen from the drug eruption: superficial perivascular infiltration of lymphocytes with prominent eosinophils.

\section{Quantitative Analysis of Leukocytes}

To make the differential diagnosis between drug eruption and recurrence of $\mathrm{MF}$, immunohistochemical staining of CD3, CD4, CD8, Foxp3, as well as CD163, was performed, and the percentage of IHC-positive cells per all tumor-infiltrating cells was quantitatively analyzed by BZ-X800 (KEYENCE, Tokyo, Japan), as we previously reported [3, 4]. The 
Fig. 3. Quantitative analysis of $\mathrm{CD}^{+}$ cells, $\mathrm{CD}^{+}$cells, $\mathrm{CD}^{+}$cells, Foxp $3^{+}$cells, and $\mathrm{CD} 163^{+}$cells: the IHC-positive cells within the lymphocyte fraction and the percentage of IHC-positive cells per all tumor-infiltrating cells were automatically counted using a BZ-X800.

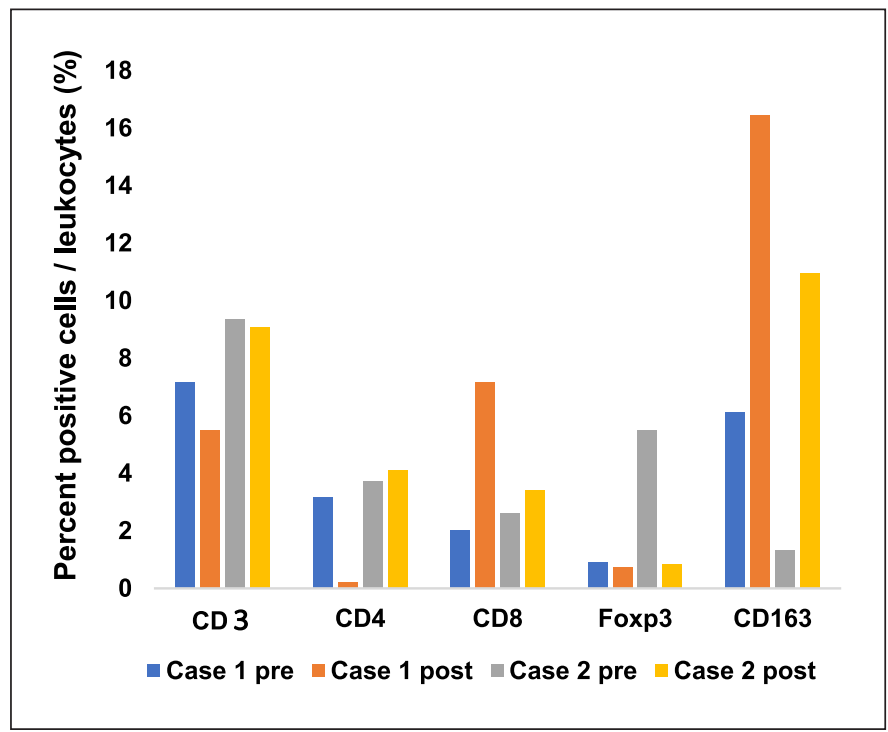

proportion of $\mathrm{CD}^{+}$cells and $\mathrm{CD} 163^{+}$cells among the leukocytes in the dermis was increased at the onset of the drug eruption in both cases; whereas, there were no increases in CD3 ${ }^{+}$ cells, $\mathrm{CD}^{+}$cells, and Foxp $3^{+}$cells among the leukocytes in the dermis (Fig. 3).

\section{Discussion}

Bexarotene is a third-generation retinoid $\mathrm{X}$ receptor-selective retinoid that has been approved for use in the treatment of CTCLs [2, 3, 5-8]. Since the objective response rate of bexarotene is relatively high, with no racial differences, bexarotene can be administered to patients with phototherapy-resistant early CTCL as one of the first-line therapies in real-world clinical practice [2,3]. In addition, several clinical trials and multicenter, retrospective studies suggested that bexarotene causes characteristic adverse events such as hypothyroidism, hyperlipidemia, and leukopenia [2, 3, 5-8]. Although various adverse events have been reported, there have been no reports of drug eruptions caused by bexarotene, even in real-world clinical experience data from post-marketing surveillance [4]. One of the possible reasons is that it is difficult to distinguish a drug eruption from recurrence of CTCL, histologically.

Although flow cytometers are widely used for diagnosing CTCL subtypes, they are difficult to use if the number of infiltrating cells is limited, such as the early stage of MF and drug eruptions. Digital microscopic analysis is useful for quantitative analysis of immunohistochemical staining, especially to identify the profiles of lymphocytes such as TILs [9, 10]. For example, we previously evaluated TILs in metastatic melanoma, the profile of which is dramatically changed by the administration of nivolumab plus ipilimumab combined therapy [10]. These reports suggested that digital microscopic analysis is even useful for the analysis of profiles of CTCL instead of flow cytometry. Indeed, in the present cases, digital microscopic analysis showed increased ratios of $\mathrm{CD}^{+}$and $\mathrm{CD} 163^{+}$cells, suggesting that the developed eruption was a drug eruption caused by bexarotene rather than recurrence of CTCL. Since the present cases were both CD4-dominant CTCLs, digital microscopic analysis was useful to make the differential diagnosis of drug eruption, which should show CD8-dominant lymphocyte infiltration. 


\section{Conclusion}

This report presents only 2 cases, but further cases may provide fundamental insights into the usefulness of digital microscopic analysis for the diagnosis of drug eruptions in CTCL patients.

\section{Statement of Ethics}

Written, informed consent was obtained from the patients for publication of this case report and any accompanying images. The protocol for this human study was approved by the Ethics Committee of Tohoku University Graduate School of Medicine, Sendai, Japan (permit no. 2019-1-308).

\section{Conflict of Interest Statement}

The authors have no conflicting interests to declare.

\section{Funding Sources}

This study was supported in part by the Japan Agency for Medical Research and Development (18lm0203062h0001, 21ym0126041h0001).

\section{Author Contributions}

T. Fujimura designed the research study. H. Chiba, Y. Kambayashi, E. Tamabuchi, K. Ohuchi, R. Amagai, A. Hasimoto, and T. Fujimura treated the patient and acquired the clinical data. K. Ohuchi performed immunohistochemical staining and its analysis. H. Chiba and T. Fujimura wrote the manuscript. T. Fujimura supervised the study.

\section{Data Availability Statement}

All data generated or analyzed during this study are included in this article. Further inquiries can be directed to the corresponding author.

\section{References}

1 Duvic M, Hymes K, Heald P, Breneman D, Martin AG, Myskowski P, et al. Bexarotene is effective and safe for treatment of refractory advanced-stage cutaneous T-cell lymphoma: multinational phase II-III trial results. J Clin Oncol. 2001;19:2456-71.

2 Duvic M, Martin AG, Kim Y, Olsen E, Wood GS, Crowley CA, et al. Phase 2 and 3 clinical trial of oral bexarotene (Targretin capsules) for the treatment of refractory or persistent early-stage cutaneous T-cell lymphoma. Arch Dermatol. 2001;137:581-93.

3 Fujimura T, Okabe T, Tanita K, Sato Y, Lyu C, Kambayashi Y, et al. A novel technique to diagnose non-melanoma skin cancer by thermal conductivity measurements: correlations with cancer stromal factors. Exp Dermatol. 2019;28: 1029-35.

4 Fujimura T, Kambayashi Y, Sato Y, Tanita K, Amagai R, Hashimoto A, et al. Successful treatment of unresectable advanced melanoma by administration of nivolumab with ipilimumab before primary tumor resection. Front Med. 2019;6:140.

\section{Karger'}


5 Hamada T, Tokura Y, Sugaya M, Ohtsuka M, Tsuboi R, Nagatani T, et al. Long-term efficacy and safety of bexarotene for Japanese patients with cutaneous T-cell lymphoma: the results of a phase 2 study (B-1201). J Dermatol. 2019;46:557-63.

6 Hamada T, Sugaya M, Tokura Y, Ohtsuka M, Tsuboi R, Nagatani T, et al. Phase I/II study of the oral retinoid X receptor agonist bexarotene in Japanese patients with cutaneous T-cell lymphomas. J Dermatol. 2017;44: 135-42.

7 Fujimura T, Sato Y, Tanita K, Amagai R, Shimauchi T, Ogata D, et al. Case series of cutaneous T-cell lymphomas treated with bexarotene-based therapy. J Dermatol. 2020;47:636-40.

8 Fujimura T, Amagai R, Kambayashi Y, Aiba S. Topical and systemic formulation options for cutaneous T cell lymphomas. Pharmaceutics. 2021;13(2): 200.

9 Mehta-Shah N, Horwitz SM, Ansell S, Ai WZ, Barnes J, Barta SK, et al. NCCN guidelines insights: primary cutaneous lymphomas, version 2.2020. J Natl Compr Canc Netw. 2020;18:522-36.

10 Hamada T, Morita A, Suga H, Boki H, Fujimura T, Hirai Y, et al. Safety andefficacy of bexarotene for Japanese patients with cutaneous T-cell lymphoma: real-world experience from post-marketing surveillance. J Dermatol. 2021. Epub ahead of print. 